\title{
Models of phase stability in Jackiw-Teitelboim gravity
}

\author{
Arindam Lala* \\ Instituto de Física, Pontificia Universidad Católica de Valparaíso, Casilla, 4059 Valparaiso, Chile \\ Dibakar Roychowdhury ${ }^{\dagger}$ \\ Department of Physics, Indian Institute of Technology Roorkee, Roorkee, 247667 Uttarakhand, India
}

(Received 4 October 2019; published 31 December 2019)

\begin{abstract}
We construct solutions within Jackiw-Teitelboim (JT) gravity in the presence of nontrivial couplings between the dilaton and the Abelian 1-form where we analyze the asymptotic structure as well as the phase stability corresponding to charged black hole solutions in $1+1 \mathrm{D}$. We consider the Almheiri-Polchinski model as a specific example within $1+1 \mathrm{D}$ JT gravity which plays a pivotal role in the study of Sachdev-YeKitaev (SYK)/anti-de Sitter (AdS) duality. The corresponding vacuum solutions exhibit a rather different asymptotic structure than their uncharged counterpart. We find interpolating vacuum solutions with $\mathrm{AdS}_{2}$ in the IR and Lifshitz 2 in the UV with dynamical exponent $z_{\text {dyn }}=3 / 2$. Interestingly, the presence of charge also modifies the black hole geometry from asymptotically AdS to asymptotically Lifshitz with the same value of the dynamical exponent. We consider specific examples, where we compute the corresponding free energy and explore the thermodynamic phase stability associated with charged black hole solutions in $1+1 \mathrm{D}$. Our analysis reveals the existence of a universal thermodynamic feature that is expected to reveal its immediate consequences on the dual SYK physics at finite density and strong coupling.
\end{abstract}

DOI: $10.1103 /$ PhysRevD.100.124061

\section{INTRODUCTION AND MOTIVATIONS}

For the last couple of decades, there had been considerable efforts toward a profound understanding of the underlying nonperturbative dynamics in large $N$ gauge theories using the celebrated AdS/CFT framework [1-3]. Nonetheless, to date there exist only a few examples where this duality can actually be tested with precise accuracy. In other words, one can exactly solve the spectrum on both sides of the duality albeit they are strongly interacting. In the recent years, an example of this kind has emerged where the spectrum of the $0+1$ dimensional strongly interacting Sachdev-Ye-Kitaev (SYK) model can be solved exactly using large $N$ techniques whose dual counterpart has been conjectured to be the Jackiw-Teitelboim (JT) model in $1+1$ D [4-31]. Apart from being exactly solvable, the SYK model exhibits maximal chaos together with an emergent conformal symmetry at low energies which therefore provides a reliable platform to test the holographic correspondence.

\footnotetext{
*arindam.physics1@gmail.com; arindam.lala@pucv.cl

†dibakarphys@gmail.com; dibakarfph@iitr.ac.in
}

Published by the American Physical Society under the terms of the Creative Commons Attribution 4.0 International license. Further distribution of this work must maintain attribution to the author(s) and the published article's title, journal citation, and DOI. Funded by SCOAP ${ }^{3}$.
For the last couple of years, there has been a systematic effort toward unveiling the dual gravitational counterpart of the SYK model. A hint came from the JT dilaton gravity in $1+1 \mathrm{D}$ [32-36] based on which disparate dual gravity models have been proposed [37-44] along with several interesting extensions [45-49].

The original SYK/AdS duality deals with Majorana fermions for which neutral dual gravity models are enough to consider. This has been the line of analyses for most of the models so far. However, very recently charged SYK models have been constructed in $[50,51]$ whose dual gravitational counterpart has been proposed to be given by the $2 \mathrm{D}$ effective gravity action of the following form ${ }^{1}$ [50]:

$S_{2 \mathrm{D}} \sim \int d^{2} x \sqrt{-g}\left(\Phi^{2} \mathcal{R}+V\left(\Phi^{2}\right)-\frac{Z\left(\Phi^{2}\right)}{4} F^{\mu \nu} F_{\mu \nu}\right)$.

The last term in the above action (1) represents the nontrivial coupling between the dilaton and the Abelian 1-form. This interaction term can be viewed as an effective coupling which can be obtained as a result of dimensional reduction from the $3+1 \mathrm{D}$ version of the theory [50]. Equation (1) without the gauge field is precisely the form of the action considered in [37] with the potential $V\left(\Phi^{2}\right)$ linear in dilaton. In the present analysis, we choose to work

\footnotetext{
${ }^{1}$ See Appendix A for details.
} 
with two specific forms of the dilaton coupling, namely, $Z\left(\Phi^{2}\right) \sim\left(\Phi^{2}\right)^{2}$ and $Z\left(\Phi^{2}\right) \sim e^{-\Phi^{2}}$ together with the choice of the dilaton potential $V\left(\Phi^{2}\right)$ as given in [37].

Based on the classical gravity computations, we construct charged 2D black hole solutions in the two aforementioned models. Our analysis reveals that the presence of charge substantially modifies the asymptotic symmetries of the space-time, namely, converting it to a two-dimensional asymptotic Lifshitz geometry which otherwise would have been an $\mathrm{AdS}_{2}$ geometry. We further compute the free energy and explore the thermodynamic phase stability of the obtained solutions. For both the models, we observe a universal thermodynamic feature of phase stability at sufficiently low temperatures and finite density.

The organization of the paper is as follows: In Sec. II, we propose our first model with $Z\left(\Phi^{2}\right) \sim\left(\Phi^{2}\right)^{2}$. Considering linear potential for the dilaton potential we explore the bounds on the potential which makes the space-time asymptotically AdS. In Secs. III and IV we comment on the vacuum structures as well as the black hole solutions both for an asymptotically flat and asymptotically AdS space-time, respectively. In Sec. V we construct perturbative solutions (in charge, $Q$ ) to our model and analyze the underlying geometry associated to both the vacuum and as well as the charged black hole solutions. This is supplemented with the study of the phase stability of derived solutions using the standard background subtraction method [52]. In Sec. VI we repeat our analysis for the exponential dilaton coupling. Finally, we conclude in Sec. VII where we mention the possible implications of our findings on the corresponding SYK counterpart.

\section{EXAMPLE I: QUADRATIC COUPLING}

We start with the Einstein-Maxwell-dilaton action of the following form:

$$
\begin{aligned}
S= & -\int d^{2} x \sqrt{-g}\left(\mathcal{R} \Phi^{2}-U\left(\Phi^{2}\right)-\frac{1}{4}\left(\Phi^{2}\right)^{2} F^{2}\right) \\
& -\int d t \sqrt{-\gamma} \Phi^{2} \mathcal{K},
\end{aligned}
$$

where $F=d A$ is the Maxwell 2-form field, $\Phi$ is the dilaton and $U\left(\Phi^{2}\right)$ is the dilaton potential. Notice that we have added the Gibbons-Hawking-York boundary term [53,54] in the above action, where $\gamma$ is the determinant of the induced metric on the boundary and $\mathcal{K}$ is the trace of the extrinsic curvature [55]. In the subsequent analysis we set the AdS length scale $L=1$ and $16 \pi G=1$.

The equations of motion can be written as

$$
\begin{aligned}
0= & \left(\nabla_{\mu} \nabla_{\nu}-g_{\mu \nu} \square\right) \Phi^{2}+\frac{1}{2} \Phi^{4}\left(F_{\mu \rho} F_{\nu}{ }^{\rho}-\frac{1}{4} F^{2} g_{\mu \nu}\right) \\
& -\frac{1}{2} g_{\mu \nu} U\left(\Phi^{2}\right),
\end{aligned}
$$

$$
\begin{gathered}
0=\mathcal{R}-\frac{F^{2}}{2} \Phi^{2}-\frac{\partial U\left(\Phi^{2}\right)}{\partial \Phi^{2}} \\
0=\partial_{\mu}\left(\sqrt{-g} \Phi^{4} F^{\mu \nu}\right)
\end{gathered}
$$

Let us now consider the conformal gauge

$$
d s^{2}=-e^{2 \omega\left(x^{+}, x^{-}\right)} d x^{+} d x^{-},
$$

where $x^{ \pm}=t \pm z$. In this light-cone gauge the equations of motion can be written as

$$
\begin{gathered}
4 \partial_{+} \partial_{-} \Phi^{2}-e^{2 \omega} U\left(\Phi^{2}\right)-2 \Phi^{4} e^{-2 \omega} F_{+-}^{2}=0, \\
\partial_{ \pm} \partial_{ \pm} \Phi^{2}-2\left(\partial_{ \pm} \omega\right)\left(\partial_{ \pm} \Phi^{2}\right)=0, \\
4 \partial_{+} \partial_{-} \omega-\frac{e^{2 \omega}}{2} \frac{\partial U\left(\Phi^{2}\right)}{\partial \Phi^{2}}+2 e^{-2 \omega} \Phi^{2} F_{+-}^{2}=0, \\
\partial_{ \pm} \chi=0,
\end{gathered}
$$

where we have defined $\chi=\sqrt{-g} \Phi^{4} F^{+-}$. Notice that our analysis differs from that of [37] in the sense that there is no fully decoupled equation of motion for $\omega(z)$. As a result we obtain a different solution for the conformal factor $e^{2 \omega(z)}$.

In the next step we would like to consider the static solutions. In order to do so, we revert back to the $(t, z)$ coordinates and use the following ansatz for the gauge field:

$A_{+}=\frac{1}{2}\left(A_{t}+A_{z}\right), \quad A_{-}=\frac{1}{2}\left(A_{t}-A_{z}\right), \quad A_{\mu}=\left(A_{t}, 0\right)$.

In these coordinates (5a)-(5d) can be expressed as

$$
\begin{gathered}
\left(\Phi^{2}\right)^{\prime \prime}+e^{2 \omega} U\left(\Phi^{2}\right)+\frac{1}{2} \Phi^{4} e^{-2 \omega} A_{t}^{\prime 2}=0 \\
\left(\Phi^{2}\right)^{\prime \prime}-2 \omega^{\prime}\left(\Phi^{2}\right)^{\prime}=0 \\
2 \omega^{\prime \prime}+e^{2 \omega} \frac{\partial U\left(\Phi^{2}\right)}{\partial \Phi^{2}}-e^{-2 \omega} \Phi^{2} A_{t}^{\prime 2}=0 \\
\Phi^{2} A_{t}^{\prime \prime}-2 A_{t}^{\prime}\left(\Phi^{2} \omega^{\prime}-\left(\Phi^{2}\right)^{\prime}\right)=0 .
\end{gathered}
$$

We can rewrite (7a) in the following form:

$$
U\left(\Phi^{2}\right)=-e^{-2 \omega}\left(\Phi^{2}\right)^{\prime \prime}-\frac{1}{2} \Phi^{4} e^{-4 \omega} A_{t}^{\prime 2} .
$$

Let us now consider a constant dilaton profile: $\Phi^{2}(z)=\Phi_{0}^{2}$. In this case substituting (8) in (7c) we obtain 


$$
2 \omega^{\prime \prime}=-\left.2 e^{2 \omega} \frac{\partial U\left(\Phi^{2}\right)}{\partial \Phi^{2}}\right|_{\Phi^{2}=\Phi_{0}^{2}} .
$$

The Ricci scalar $(\mathcal{R})$, on the other hand, can be written as

$$
\mathcal{R}=\left.2 \frac{\partial U\left(\Phi^{2}\right)}{\partial \Phi^{2}}\right|_{\Phi^{2}=\Phi_{0}^{2}}
$$

where we have used (9).

Now, in order for the space-time to have $\mathrm{AdS}_{2}$ asymptotics we must have the following criteria:

$$
\left.\frac{\partial U\left(\Phi^{2}\right)}{\partial \Phi^{2}}\right|_{\Phi^{2}=\Phi_{0}^{2}}<0
$$

\section{GRAVITY IN ASYMPTOTICALLY FLAT 1 + 1 D}

\section{A. Minkowski vacuum}

In this section, we would like to consider the vacuum solution corresponding to $1+1 \mathrm{D}$ dilaton gravity. In order to do so we consider the following metric:

$$
d s^{2}=-d t^{2}+d z^{2}
$$

From the gauge equation of motion (3c) we can write

$$
F_{t z}=\frac{Q}{\Phi^{4}}
$$

It is easy to check that the scalar equation of motion (3b) and one of the trace equations of motion corresponding to the metric, (3a), lead to the following form of the dilaton potential:

$$
U\left(\Phi^{2}\right)=-\frac{1}{2} \frac{Q^{2}}{\Phi^{4}}
$$

On the other hand, the remaining trace equation of motion can be written expressed as

$$
\left(\Phi^{2}\right)^{\prime \prime}+\frac{1}{4} \frac{Q^{2}}{\Phi^{4}}+\frac{1}{2} U\left(\Phi^{2}\right)=0
$$

Substituting (14) into (15) we obtain

$$
\left(\Phi^{2}\right)^{\prime \prime}=0
$$

whose solution may be written as

$$
\Phi^{2}=b z+a
$$

where $a$ and $b$ are arbitrary integration constants.

\section{B. Charged black hole solutions}

We choose to work with metric ansatz of the following form:

$$
d s^{2}=-f(z) d t^{2}+f^{-1}(z) d z^{2}
$$

The only component of the Maxwell field strength tensor can thus be written as

$$
F_{t z}=\frac{Q}{\Phi^{4}(z)}
$$

where $Q$ is an integration constant which we can identify as the charge of the black hole, and we have used (3c) in order to derive (19).

With the metric (18) the remaining equations of motion (3a) and (3b) can be written as

$$
\begin{gathered}
f^{\prime}(z)\left(\Phi^{2}\right)^{\prime}+\frac{Q^{2}}{2\left(\Phi^{2}\right)^{2}}+U\left(\Phi^{2}\right)=0, \\
2 f(z)\left(\Phi^{2}\right)^{\prime \prime}+f^{\prime}(z)\left(\Phi^{2}\right)^{\prime}+\frac{Q^{2}}{2\left(\Phi^{2}\right)^{2}}+U\left(\Phi^{2}\right)=0, \\
f^{\prime \prime}(z)-\frac{Q^{2}}{\Phi^{6}(z)}+\frac{\partial U\left(\Phi^{2}\right)}{\partial \Phi^{2}}=0 .
\end{gathered}
$$

From (20) and (21) it is easy to check that

$$
\left(\Phi^{2}\right)^{\prime \prime}=0
$$

and as a result the dilaton becomes constant at the boundary $z=0$.

As a next step, we determine the metric coefficient $f(z)$ with particular choices of the dilaton potential $U\left(\Phi^{2}\right)$ where we finally set $\Phi^{2}=\varphi_{0}=$ const.

\section{Case I: The Almheiri-Polchinski model [37]} $\left[U\left(\Phi^{2}\right)=C-A \Phi^{2}\right]$ and the Callan-Giddings-HarveyStrominger (CGHS) model [56] [U( $\left.\left.\Phi^{2}\right)=-A \Phi^{2}\right]$

From (22) we obtain

$$
f^{\prime \prime}(z)=A+\frac{Q^{2}}{\varphi_{0}^{3}},
$$

whose solution may be written as

$$
f(z)=\left(1-\frac{z}{z_{H}}\right)\left[1-\frac{1}{2}\left(A+\frac{Q^{2}}{\varphi_{0}^{3}}\right) z z_{H}\right]
$$

Using (25), the Hawking temperature of the black hole can be obtained as 


$$
\begin{aligned}
T_{H} & =\left.\frac{1}{4 \pi} \partial_{z} \sqrt{-\frac{g_{t t}}{g_{z z}}}\right|_{z=z_{H}} \\
& =\frac{1}{4 \pi z_{H}}\left[\frac{1}{2}\left(A+\frac{Q^{2}}{\varphi_{0}^{3}}\right) z_{H}^{2}-1\right] .
\end{aligned}
$$

The extremal limit, in which the Hawking temperature vanishes, is characterized by the extremal value of the charge given by

$$
Q_{e}^{2}=\left(\frac{2}{z_{H}^{2}}-A\right) \varphi_{0}^{3}
$$

On the other hand, by using the Wald formalism, the entropy of the black hole is given by [57]

$$
S_{W}=4 \pi \Phi^{2}
$$

The thermodynamic stability of the black hole is determined by computing the corresponding heat capacity $(C)$ :

$$
C=T_{H} \frac{\partial S_{W}}{\partial T_{H}}=\frac{8 \pi \varphi_{0}^{4}}{3 Q^{2} z_{H}^{2}}\left[1-\frac{1}{2}\left(A+\frac{Q^{2}}{\varphi_{0}^{3}}\right) z_{H}^{2}\right],
$$

where we have used (26) and (28).

For nonextremal black holes one must have $T_{H}>0$ which leads to the following condition:

$$
\frac{1}{2}\left(A+\frac{Q^{2}}{\varphi_{0}^{3}}\right) z_{H}^{2}>1
$$

Substituting (30) in (29) we note that the specific heat is always negative. This suggests that the black holes (25) in an asymptotically flat space-time are indeed unstable and decay through Hawking radiation.

\section{Case II: Magnetic branes [58], $U\left(\Phi^{2}\right)=\frac{B^{2}}{\Phi^{2}}-A \Phi^{2}$}

The equation of motion corresponding to the metric $f(z)$ can be expressed as

$$
f^{\prime \prime}(z)=A+\frac{B}{\varphi_{0}^{2}}+\frac{Q^{2}}{\varphi_{0}^{3}},
$$

where we have used (22). The general solution to the above equation (31) is given by

$$
f(z)=\left(1-\frac{z}{z_{H}}\right)\left[1-\frac{1}{2}\left(A+\frac{B}{\varphi_{0}^{2}}+\frac{Q^{2}}{\varphi_{0}^{3}}\right) z z_{H}\right] .
$$

Proceeding in the same line of analysis as in the previous case I, the thermodynamic quantities may be found as follows: (i) the Hawking temperature

$$
T_{H}=\frac{1}{4 \pi z_{H}}\left[\frac{1}{2}\left(A+\frac{B}{\varphi_{0}^{2}}+\frac{Q^{2}}{\varphi_{0}^{3}}\right) z_{H}^{2}-1\right],
$$

(ii) extremal value of charge

$$
Q_{e}^{2}=\left[\frac{2}{z_{H}^{2}}-\left(A+\frac{B}{\varphi_{0}^{2}}\right)\right] \varphi_{0}^{3},
$$

(iii) the specific heat

$$
\begin{aligned}
C & =T_{H} \frac{\partial S_{W}}{\partial T_{H}} \\
& =\frac{4 \pi \varphi_{0}^{3}}{z_{H}^{2}}\left[1-\frac{1}{2}\left(A+\frac{B}{\varphi_{0}^{2}}+\frac{Q^{2}}{\varphi_{0}^{3}}\right) z_{H}^{2}\right]\left(B+\frac{3 Q^{2}}{2 \varphi_{0}}\right)^{-1} .
\end{aligned}
$$

Like in the previous example, it is easy to check that the condition for extremality implies the thermodynamic instability in black holes.

\section{VACUUM SOLUTIONS WITH AdS ASYMPTOTICS}

Unlike the previous example, here we discuss the possibilities on vacuum solutions with $\mathrm{AdS}_{2}$ asymptotics. We show that solutions with $\mathrm{AdS}_{2}$ asymptotics are indeed possible both for the constant as well as the running dilaton profiles.

\section{A. Solution with constant dilaton}

We first construct solutions with constant dilaton $\Phi^{2}=\Phi_{0}^{2}$

\section{Case I: $e^{2 \omega}=\frac{1}{z^{2}}$}

In this case from (7a) we may write

$$
A_{t}^{\prime 2}=-\frac{2}{z^{4} \Phi_{0}^{4}} U\left(\Phi_{0}^{2}\right)
$$

whereas (7b) is satisfied trivially. Notice that in the above equation $U\left(\Phi_{0}^{2}\right)$ must be negative. Using the relation $\omega^{\prime}=-\frac{1}{z}$ we can write $(7 \mathrm{c})$ as

$$
\frac{2}{z^{2}}+\left.\frac{1}{z^{2}} \frac{\partial U\left(\Phi^{2}\right)}{\partial \Phi^{2}}\right|_{\Phi_{0}^{2}}-z^{2} \Phi_{0}^{2} A_{t}^{\prime 2}=0 .
$$

Finally, using (36) we obtain the following relation:

$$
\left.\frac{\partial U\left(\Phi^{2}\right)}{\partial \Phi^{2}}\right|_{\Phi_{0}^{2}}=-2\left(1-\frac{\left|U\left(\Phi_{0}^{2}\right)\right|}{\Phi_{0}^{2}}\right)
$$

which leads to the following bound to the potential: 


$$
\frac{\left|U\left(\Phi_{0}^{2}\right)\right|}{\Phi_{0}^{2}}<1
$$

\section{Case II: $e^{2 \omega}=\frac{1}{\sinh ^{2} z}$}

In this case the equation for the gauge field can be expressed as

$$
A_{t}^{\prime 2}=-\frac{2}{\Phi_{0}^{4} \sinh ^{4} z} U\left(\Phi_{0}^{2}\right),
$$

from which we again conclude that $U\left(\Phi_{0}^{2}\right)<0$. Substituting $e^{2 \omega}$ in (7c) it is trivial to check that one arrives at the same constraint conditions (38) and (39).

\section{Case III: $e^{2 \omega}=\frac{1}{\sin ^{2} z}$}

In this case the equation for the gauge field can be written as

$$
A_{t}^{\prime 2}=-\frac{2}{\Phi_{0}^{4} \sin ^{4} z} U\left(\Phi_{0}^{2}\right)
$$

from which it is evident that $U\left(\Phi_{0}^{2}\right)<0$. Substituting $e^{2 \omega}$ in $(7 \mathrm{c})$ we once again arrive at the same constraint conditions (38) and (39).

Thus we observe that the above constraint conditions (38) and (39) are universal for all three cases considered; i.e., they are the same irrespective of the choice of the coordinate systems.

\section{B. Solutions with running dilaton}

Let us consider the metric of the following form:

$$
d s^{2}=\frac{1}{z^{2}}\left(-d t^{2}+d z^{2}\right) .
$$

With this choice of metric the gauge equation of motion $(3 \mathrm{c})$ leads to

$$
F_{t z}=\frac{Q}{\Phi^{4} z^{2}} .
$$

The metric equation of motion can be expressed as

$$
\begin{gathered}
z\left(\Phi^{2}\right)^{\prime}-\frac{1}{4} \frac{Q^{2}}{\Phi^{4}}-\frac{1}{2} U\left(\Phi^{2}\right)=0, \\
z^{2}\left(\Phi^{2}\right)^{\prime \prime}+z\left(\Phi^{2}\right)^{\prime}+\frac{1}{4} \frac{Q^{2}}{\Phi^{4}}+\frac{1}{2} U\left(\Phi^{2}\right)=0 .
\end{gathered}
$$

Substituting (44) into (45) we obtain

$$
z^{2}\left(\Phi^{2}\right)^{\prime \prime}+2 z\left(\Phi^{2}\right)^{\prime}=0,
$$

whose solution may be formally expressed as

$$
\Phi^{2}=-\frac{b_{1}}{z}+a_{1}
$$

It is interesting to note that the dilaton diverges near the boundary, $z \approx 0$.

In a similar way, considering the conformal factor as $e^{2 \omega}=\sinh ^{-2} z, \sin ^{-2} z$, the solutions to the dilaton can be found as

$$
\begin{aligned}
& \Phi^{2}=-b_{2} \operatorname{coth} z+a_{2}, \\
& \Phi^{2}=-b_{3} \cot z+a_{3},
\end{aligned}
$$

respectively. Interestingly, both these solutions diverge near the boundary, $z \approx 0$.

Notice that the divergence of the dilaton profile (47)-(49) near the boundary $(z \approx 0)$ is a generic feature of the AdS space-time; see for example [59] and references therein. However, as far as the present analysis is concerned, one of the solutions (47) has an interesting consequence from the perspective of the SYK/AdS duality. One of the interesting facets of this duality is that it allows us to relate the SYK degrees of freedom to the underlying dynamics of the $\mathrm{AdS}_{2}$ counterpart, [11,42-44]. We can consistently set $a_{1}=0$ in (47) by demanding that the dilaton $\Phi^{2}$ vanishes as we probe deep IR $(z \rightarrow \infty)$. This observation is in fact consistent with the strongly interacting $\left(J \sim 1 / \Phi^{2} \gg 1\right)$ nature of the dual SYK model in which we are mostly interested [42-44].

\section{GENERAL SOLUTIONS: A PERTURBATIVE APPROACH}

In this section, we adopt perturbation techniques in order to find the general solutions to the equations of motion (3a)-(3c) and determine the metric of the space-time. In order to perform our analysis, we consider a running dilaton where the dilaton is a function of $z$ only: $\Phi^{2}=$ $\Phi^{2}(z)$. We also consider the dilaton potential as ${ }^{2}$ [37]

$$
U\left(\Phi^{2}\right)=C-A \Phi^{2}, \quad C, A>0 .
$$

In order to obtain solutions to the metric as well as the dilaton equations of motion we expand the above entities as a perturbation in the $U(1)$ charge $Q$, namely,

$$
\begin{gathered}
\Phi^{2}(z)=\Phi_{(0)}^{2}(z)+Q^{2} \Phi_{(1)}^{2}(z)+\cdots, \\
\omega(z)=\omega_{(0)}(z)+Q^{2} \omega_{(1)}(z)+\cdots .
\end{gathered}
$$

\footnotetext{
${ }^{2}$ See Appendix $B$ regarding the black hole solution with $A<0$.
} 
The Maxwell field strength tensor is given by the solution of (7d) which may be written as

$$
F_{z t} \equiv A_{t}^{\prime}=-\frac{Q e^{2 \omega}}{\Phi^{4}(z)} .
$$

On the other hand, the equation of motion for the dilaton (7b) can be recast in the following form:

$$
\left(\Phi^{2}\right)^{\prime}=\tilde{C} e^{2 \omega},
$$

whose solution may be written as

$$
\Phi^{2}(z)=\tilde{C} \int e^{2 \omega} d z+\bar{C},
$$

where $\tilde{C}$ and $\bar{C}$ are arbitrary integration constants.

In the following we note down the equations of motion up to leading order in the perturbative expansion. Substituting (51b) into (7c) we find

$$
\begin{gathered}
\mathcal{O}\left(Q^{0}\right): 0=\omega_{(0)}^{\prime \prime}-e^{2 \omega_{(0)}} \\
\mathcal{O}\left(Q^{2}\right): 0=2 \omega_{(1)}^{\prime \prime}-4 e^{2 \omega_{(0)}} \omega_{(1)}-e^{2 \omega_{(0)}}\left(\Phi_{(0)}^{2}\right)^{-3} .
\end{gathered}
$$

On the other hand, substituting (51a) into (7a) we find

$$
\begin{aligned}
& \mathcal{O}\left(Q^{0}\right): 0=\left(\Phi_{(0)}^{2}\right)^{\prime \prime}+2 e^{2 \omega_{(0)}}\left(1-\Phi_{(0)}^{2}\right), \\
& \mathcal{O}\left(Q^{2}\right): 0=\left(\Phi_{(1)}^{2}\right)^{\prime \prime}-2 e^{2 \omega_{(0)}} \Phi_{(1)}^{2} \\
&+4 e^{2 \omega_{(0)}}\left[\omega_{(1)}\left(1-\Phi_{(0)}^{2}\right)+\frac{1}{8}\left(\Phi_{(0)}^{2}\right)^{-2}\right] .
\end{aligned}
$$

\section{A. Interpolating vacuum solutions}

The vacuum solution corresponding to $Q=0$ is characterized by [37]

$$
\begin{gathered}
e^{2 \omega_{(0)}^{\mathrm{vac}}(z)}=\frac{1}{z^{2}}, \\
\Phi_{(0)}^{2^{\mathrm{vac}}}(z)=\left(1+\frac{1}{2 z}\right),
\end{gathered}
$$

which satisfy the zeroth-order equations (55) and (57), respectively, for the following values of the constants in (54): $\tilde{C}=-1 / 2$ and $\bar{C}=1$. Thus for the purpose of our present analysis it is sufficient to solve the $\mathcal{O}\left(Q^{2}\right)$ equations of motion, namely, (56) and (58).

Using (56) the solution corresponding to $\omega_{(1)}^{\mathrm{vac}}$ can be expressed as ${ }^{3}$

\footnotetext{
${ }^{3} \mathrm{We}$ set the second integration constant $\mathrm{C}_{3}$ to zero. This is to render the on-shell action in Sec. V C finite at the horizon.
}

$\omega_{(1)}^{\mathrm{vac}}(z)=\frac{\mathrm{C}_{4}}{z}+\frac{1-2 z-4 z^{2}+2(1+2 z) \cdot \log (1+2 z)}{8 z(1+2 z)}$.

Thus the metric for the vacuum can be expressed as

$$
d s^{2}=e^{2 \omega_{(0)}}\left(1+2 Q^{2} \omega_{(1)}^{\mathrm{vac}}\right)\left(-d t^{2}+d z^{2}\right) .
$$

Let us now analyse the IR and UV behaviors of the solution (62).

(i) In the IR region $z \rightarrow \infty$ the behavior of the metric is given by

$$
e^{2 \omega} \simeq \frac{1}{z^{2}}\left(1-\frac{Q^{2}}{2}\right)+\mathcal{O}\left(z^{-3}\right)
$$

which thereby leads to an emerging $\mathrm{AdS}_{2}$ geometry.

(ii) The UV $(z=0)$ behavior of the metric is found to be of the following form:

$$
e^{2 \omega} \simeq \frac{Q^{2}}{4 z^{3}}\left(1+8 \mathrm{C}_{4}\right)+\frac{1}{z^{2}}-\frac{2 Q^{2}}{3}+\mathcal{O}(z),
$$

whose leading contribution comes from the first term on the rhs of (64). This turns out to be a Lifshitz 2 geometry with dynamical exponent $z_{\mathrm{dyn}}=\frac{3}{2}$.

Thus we observe that the vacuum solution interpolates between Lifshitz $_{2}$ in the UV and $\mathrm{AdS}_{2}$ in the deep IR. It is interesting to note that in the absence of the charge, $Q=0$, the geometry is $\mathrm{AdS}_{2}$ for both in the IR as well as the UV. Thus we conclude that the presence of the gauge field modifies the UV asymptotics from $\mathrm{AdS}_{2}$ to Lifshitz 2 [60].

\section{B. Charged 2D black holes}

The zeroth-order solutions $\Phi_{(0)}^{2}(z)$ and $\omega_{(0)}(z)$ are, respectively, given by [37]

$$
\begin{gathered}
\Phi_{(0)}^{2}=1+\sqrt{\mu} \operatorname{coth}(2 \sqrt{\mu} z), \\
e^{2 \omega_{(0)}}=\frac{4 \mu}{\sinh ^{2}(2 \sqrt{\mu} z)} .
\end{gathered}
$$

Notice that, in order for the solution (65a) to be consistent with the equations of motion (7a) and (7b), we must choose the constants appearing in (54) as $\tilde{C}=-1 / 2$ and $\bar{C}=1$.

It is trivial to check that, with the choice of the constants $\tilde{C}$ and $\bar{C}$, (65a) and (65b) are indeed the solutions to the equations of motion (55) and (57). Using (65a) and (65b) in (56) the solution for $\omega_{(1)}^{\mathrm{BH}}$ may be written as 


$$
\begin{aligned}
\omega_{(1)}^{\mathrm{BH}}(\rho)= & \mathrm{C}_{1} \frac{\rho}{\sqrt{\mu}}+\mathrm{C}_{2}\left[\frac{\rho}{2 \sqrt{\mu}} \cdot \log \left(\frac{\sqrt{\mu}+\rho}{\sqrt{\mu}-\rho}\right)-1\right] \\
& +\frac{1}{8 \mu^{3 / 2}(\mu-1)^{2}} \times\left\{-2 \sqrt{\mu}\left(1-\mu \frac{2+\rho}{1+\rho}+\frac{\mu^{2}}{1+\rho}\right)+4 \mu^{3 / 2} \rho \cdot \log (1+\rho)\right. \\
& \left.-\rho \cdot \log (\rho-\sqrt{\mu})\left(1-3 \mu+2 \mu^{3 / 2}\right)+\rho \cdot \log (\rho+\sqrt{\mu})\left(1-3 \mu-2 \mu^{3 / 2}\right)\right\},
\end{aligned}
$$

where $\mathrm{C}_{1}$ and $\mathrm{C}_{2}$ are the integration constants and we have used the following change in the spatial coordinate $[37,45]$ :

$$
z \rightarrow \frac{1}{2 \sqrt{\mu}} \operatorname{coth}^{-1}\left(\frac{\rho}{\sqrt{\mu}}\right)
$$

In the subsequent calculations we set $\mathrm{C}_{2}=0$ in order to obtain a physically meaningful asymptotic structure of the space-time.

Finally, using (51b), (65b) and (66) the metric (4) corresponding to the black hole geometry can be expressed as

$$
\begin{aligned}
d s^{2} & =e^{2 \omega}\left(-d t^{2}+d z^{2}\right) \\
& =\frac{4 \mu}{\sinh ^{2} 2 \sqrt{\mu} z}\left(1+2 Q^{2} \omega_{(1)}^{\mathrm{BH}}(z)\right)\left(-d t^{2}+d z^{2}\right) \\
& =4\left(\rho^{2}-\mu\right)\left(1+2 Q^{2} \omega_{(1)}^{\mathrm{BH}}(\rho)\right)\left(-d t^{2}+\frac{d \rho^{2}}{4\left(\rho^{2}-\mu\right)^{2}}\right) .
\end{aligned}
$$

Notice that the above black hole solution (67) has a horizon at $\rho=\sqrt{\mu}$. On the other hand, the boundary is located at $\rho=\infty$. Expanding the metric near the boundary we find

$$
e^{2 \omega(\delta)} \simeq \frac{8 Q^{2} \mathrm{C}_{1}}{\sqrt{\mu} \delta^{3}}+\frac{4}{\delta^{2}}-\frac{8 Q^{2} \mathrm{C}_{1} \sqrt{\mu}}{\delta}-4 \mu+\mathcal{O}(\delta),
$$

where we have changed the variable $\rho \rightarrow 1 / \delta$ and taken the limit $\delta \rightarrow 0$ subsequently. The leading term in the expansion (69) behaves as $\sim \frac{1}{\delta^{3}}$, which is a signature of an asymptotically Lifshitz geometry with dynamical critical exponent $z_{\text {dyn }}=\frac{3}{2}$. It is trivial to check that in the limit $Q^{2} \rightarrow 0$ the resulting metric is that of an asymptotically $\mathrm{AdS}_{2}$ space-time. Our result thus indicates that in the presence of the gauge field the asymptotic behavior of the space-time indeed changes from $\mathrm{AdS}_{2}$ to Lifshitz 2 [60].

Next, we turn our attention toward computing the dilaton profile for our model. Using (54) we observe that

$$
\begin{aligned}
\Phi_{(1)}^{2} & =-\int e^{2 \omega_{0}}(z) \omega_{(1)}(z) d z \\
& =2 \int \omega_{(1)}(\rho) d \rho .
\end{aligned}
$$

Notice that in writing the second line we have used (67). Thus the complete solution up to $\mathcal{O}\left(Q^{2}\right)$ can be expressed as

$$
\begin{aligned}
\Phi^{2} & \simeq \Phi_{(0)}^{2}+Q^{2} \Phi_{(1)}^{2} \\
& =(1+\rho)+2 Q^{2} \int \omega_{(1)}(\rho) d \rho
\end{aligned}
$$

We now compute the thermodynamic quantities corresponding to the above black hole geometry (68). The corresponding Hawking temperature is given by

$$
T_{H}=\left.\frac{1}{2 \pi} \sqrt{-\frac{1}{4} g^{t t} g^{\rho \rho}\left(\partial_{\rho} g_{t t}\right)^{2}}\right|_{\rho \rightarrow \sqrt{\mu}}=\frac{\sqrt{\mu}}{\pi}
$$

On the other hand, the Wald entropy associated with the black hole can be found as

$$
\begin{aligned}
S_{W} & =4 \pi\left[(1+\sqrt{\mu})+\left.2 Q^{2} \int \omega_{(1)}(\rho) d \rho\right|_{\rho=\sqrt{\mu}}\right] \\
& =4 \pi\left[1+\sqrt{\mu}+Q^{2} \frac{1+4 \mathrm{C}_{1} \mu|\mu-1|}{4 \sqrt{\mu}|\mu-1|}\right] .
\end{aligned}
$$

Notice that (73) is ill defined at $\mu=1$. Therefore one can define black hole solutions for either of the two branches, namely, $\mu<1$ or $\mu>1$, which is thereby consistent with the earlier observations $[37,45]$.

\section{Phase stability}

In order to check whether there is any phase transition or crossover between the empty $\mathrm{AdS}_{2}$ and the $\mathrm{AdS}_{2}$ black hole one needs to compare free energies between different configurations. We substitute (3b) into the action (2) which finally yields

$$
S=-\int d^{2} x \sqrt{-g}\left(\frac{F^{2}}{4}\left(\Phi^{2}\right)^{2}-C\right)-\int d t \sqrt{-\gamma} \mathcal{K} \Phi^{2}
$$

Next we use the equation of motion for the gauge field, (3c), to find the on-shell action as 


$$
\begin{aligned}
-S^{(\mathrm{os})}= & -\int d^{2} x \sqrt{-g} C+\int d^{2} x \partial_{\mu}\left(\sqrt{-g} F^{\mu \nu}\left(\Phi^{2}\right)^{2} A_{\nu}\right) \\
= & -C \cdot(\mathrm{Vol})+\int d t\left(\sqrt{-g} F^{z t}\left(\Phi^{2}\right)^{2} A_{t}\right) \\
& +\int d t \sqrt{-\gamma} \mathcal{K} \Phi^{2}
\end{aligned}
$$

where (Vol) is the volume term associated with the following two geometries:

(i) For empty interpolating vacuum we may write down the volume term as

$$
\begin{aligned}
(\mathrm{Vol}))_{\mathrm{Int}}^{\mathrm{vac}} & =-\int_{0}^{\beta_{0}} d \tau \int_{0}^{\Lambda} d \rho\left(\frac{1}{\rho^{2}}\right) e^{2 \omega_{\mathrm{Int}}^{\mathrm{vac}}}(\rho) \\
& =-\left.\beta_{0} \mathcal{A}_{\mathrm{vac}}(\rho)\right|_{0} ^{\Lambda},
\end{aligned}
$$

where we have used the change of coordinate $z \rightarrow \frac{1}{\rho}$ and

$$
\mathcal{A}_{\mathrm{vac}}=\rho-\frac{Q^{2} \rho}{2}\left(1-\frac{\rho}{4}\left(1+8 \mathrm{C}_{4}\right)-\frac{\rho}{2} \cdot \log \left(\frac{2+\rho}{\rho}\right)\right) .
$$

(ii) For the $\mathrm{AdS}_{2}$ black hole the volume term can be expressed as

$$
\begin{aligned}
(\mathrm{Vol})^{\mathrm{BH}} & =-\int_{0}^{\beta_{1}} d \tau \int_{\rho \sim \sqrt{\mu}}^{\rho \sim \Lambda} d \rho \frac{1}{2\left(\rho^{2}-\mu\right)} e^{2 \omega^{\mathrm{BH}}}(\rho) \\
& =-\beta_{1}\left(\mathcal{A}^{(\Lambda)}(\rho \rightarrow \Lambda)-\mathcal{A}^{(\mu)}(\rho \rightarrow \sqrt{\mu})\right),
\end{aligned}
$$

where

$$
\begin{aligned}
\mathcal{A}^{(\Lambda)}(\rho \rightarrow \Lambda)= & \frac{1}{2 \mu^{3 / 2}(\mu-1)^{2}}\{2 \Lambda \sqrt{\mu}(\mu-1)[2 \mu(\mu-1) \\
& \left.+Q^{2}\left(1+\Lambda \mathrm{C}_{1} \sqrt{\mu}(\mu-1)\right)\right] \\
& +Q^{2}\left(\Lambda^{2}-\mu\right)\left[4 \mu^{3 / 2} \cdot \log (1+\Lambda)\right. \\
& +\left(-1+3 \mu-2 \mu^{3 / 2}\right) \cdot \log (\Lambda-\sqrt{\mu}) \\
& \left.\left.+\left(1-3 \mu-2 \mu^{3 / 2}\right) \cdot \log (\Lambda+\sqrt{\mu})\right]\right\}
\end{aligned}
$$

and

$$
\mathcal{A}^{(\mu)}(\rho \rightarrow \sqrt{\mu})=\frac{4 \mu(\mu-1)+Q^{2}\left[1+4 \mu \mathrm{C}_{1}(\mu-1)\right]}{2 \sqrt{\mu}(\mu-1)} .
$$

Here, we have introduced a UV cutoff $\Lambda$ in order to make the integrals finite, and $\beta_{0}$ and $\beta_{1}$ are the periods associated with the interpolating vacuum and the $\mathrm{AdS}_{2}$ black hole, respectively. Moreover, while $\beta_{1}$ is fixed by (72), $\beta_{0}$ is arbitrary. Thus we can fix $\beta_{0}$ by demanding that, at some arbitrary $\rho=\Lambda$, the temperature of both the configurations should be the same, namely, ${ }^{4}$

$$
\frac{\beta_{0}}{\beta_{1}}=\left.\sqrt{\frac{e^{2 \omega^{\mathrm{BH}}}(\rho)}{e^{2 \omega_{\mathrm{Int}}^{\mathrm{ac}}}(\rho)}}\right|_{\rho \rightarrow \Lambda} .
$$

In the next step, we would like to compute the difference between the volume terms (78) and (76). This may be written in the following form:

$$
\begin{aligned}
\Delta(\mathrm{Vol})= & -C\left((\mathrm{Vol})^{\mathrm{BH}}-(\mathrm{Vol}) \mathrm{\textrm {Int }}\right) \\
= & C \beta_{1}\left[\left(\mathcal{A}_{1}-\mathcal{B}_{1}\right) \Lambda^{2}+\left(\mathcal{A}_{2}-\mathcal{B}_{2}\right) \Lambda\right. \\
& \left.+\left(\mathcal{A}_{3}-\mathcal{B}_{3}\right)-\mathcal{A}^{(\mu)}(\rho \rightarrow \sqrt{\mu})\right],
\end{aligned}
$$

where the individual coefficients are given by

$$
\begin{aligned}
& \mathcal{A}_{1}=\frac{2 Q^{2} \mathrm{C}_{1}}{\sqrt{\mu}}, \quad \mathcal{A}_{2}=2, \quad \mathcal{A}_{3}=\frac{Q^{2}}{2(\mu-1)}, \\
& \mathcal{B}_{1}=\frac{Q^{2}}{\sqrt{2}} \sqrt{\frac{\mathrm{C}_{1}\left(1+8 \mathrm{C}_{4}\right)}{\sqrt{\mu}},} \\
& \mathcal{B}_{2}=\frac{1}{4 \sqrt{2} \mathrm{C}_{1}} \sqrt{\frac{\mathrm{C}_{1}}{\sqrt{\mu}\left(1+8 \mathrm{C}_{4}\right)}}\left(\sqrt{\mu}\left(1+8 \mathrm{C}_{4}\right)+24 \mathrm{C}_{1}\right), \\
& \mathcal{B}_{3}=-Q^{2}\left(1+\mathrm{C}_{1} \sqrt{\mu}\right) .
\end{aligned}
$$

It is to be noted that in (84) we have simplified the cumbersome expression for $\mathcal{B}_{3}$ by using the following relation:

$$
\mathrm{C}_{1}=\frac{\sqrt{\mu}}{8}\left(1+8 \mathrm{C}_{4}\right),
$$

which is easily derived by setting the coefficient of $\Lambda^{2}$ in (82) equal to zero. In addition, using (85), it is easy to check that the coefficient of the $\Lambda$ term in (82) vanishes.

Let us now consider the second term $\Pi$ in (75). Using (52), (68) and (62) this may be written as

$$
\begin{aligned}
\Pi & =Q^{2} \int d \tau \int_{0}^{\Lambda} e^{2 \omega_{(0)}(z)}\left(\Phi_{(0)}^{2}(z)\right)^{-2} d z \\
& = \begin{cases}-\left.4 \beta_{0} Q^{2}\left(\frac{1}{2+\rho}\right)\right|_{0} ^{\Lambda} & \text { (interpolating vacuum) }, \\
-\left.2 \beta_{1} Q^{2}\left(\frac{1}{1+\rho}\right)\right|_{\sqrt{\mu}} ^{\Lambda} & \text { (black hole), }\end{cases}
\end{aligned}
$$

\footnotetext{
${ }^{4}$ The choice of normalization (81) for $\beta_{0}$ is unique in the sense that the geometry of the hypersurface placed at a radial cutoff $\rho=\Lambda(\rightarrow \infty)$ should be the same both for the vacuum as well as the black hole space-time [61]. This also ensures the consistency in the thermodynamic description in the sense of holography as the limit $\Lambda \rightarrow \infty$ is approached.
} 
where we have substituted (65a) and (65b). Finally, in the limit $\Lambda \rightarrow \infty$ the difference between the second terms can be written as

$$
\Delta(\Pi)=\Pi^{\mathrm{BH}}-\Pi^{\mathrm{vac}}=-2 Q^{2} \beta_{1}\left(\frac{1+2 \sqrt{\mu}}{1+\sqrt{\mu}}\right) .
$$

We now calculate the contribution from the third term $\Sigma$ in (75). In order to do so, we choose a $\rho=$ const hypersurface [55]. Let us now define an outward normal $n^{\rho}$, pointing along the increasing $\rho$, as

$$
g_{\rho \rho} n^{\rho} n^{\rho}=1 \Rightarrow n^{\rho}=\frac{1}{\sqrt{g_{\rho \rho}}}
$$

where $g_{\rho \rho}$ is the metric coefficient in (68) corresponding to the spatial coordinate $\rho$. The trace of the extrinsic curvature, $\mathcal{K}$, can then be written as

$$
\mathcal{K}=n^{\rho} \frac{\partial_{\rho} \sqrt{-\gamma}}{\sqrt{-\gamma}} .
$$

Next, we use (54), (88) and (89) to compute the difference between the third terms in the $\Lambda \rightarrow \infty$ limit. After a few easy steps, we note down this expression as ${ }^{5}$

$$
\begin{aligned}
\Delta \Sigma= & \Sigma^{\mathrm{BH}}-\Sigma^{\mathrm{vac}} \\
= & \frac{\beta_{1}}{8}\left(-14 Q^{2}+\mu+\frac{\mu}{Q^{4} \mathrm{C}_{1}^{2}}-\frac{\sqrt{\mu}\left(-4+4 \mu+Q^{2}\right)}{Q^{2} \mathrm{C}_{1}(\mu-1)}\right. \\
& \left.-\frac{\mu}{2 \mathrm{C}_{1}^{2} Q^{4}}-\frac{\sqrt{\mu}\left(Q^{2}-4\right)}{Q^{2} \mathrm{C}_{1}}\right) .
\end{aligned}
$$

After performing a long but simple calculation, the difference in the on-shell action can be expressed as

$$
\begin{aligned}
-\Delta S^{(\mathrm{os})}= & \beta_{1}\left[C \left\{-2 \sqrt{\mu}+Q^{2}\left(\frac{1}{2(\mu-1)}+\left(1+\mathrm{C}_{1} \sqrt{\mu}\right)\right.\right.\right. \\
& \left.\left.-\frac{\left(1+4 \mu \mathrm{C}_{1}(\mu-1)\right)}{2 \sqrt{\mu}(\mu-1)}\right)\right\}-2 Q^{2}\left(\frac{1+2 \sqrt{\mu}}{1+\sqrt{\mu}}\right) \\
& +\frac{1}{8}\left(-14 Q^{2}+\mu+\frac{\mu}{Q^{4} \mathrm{C}_{1}^{2}}-\frac{\sqrt{\mu}\left(-4+4 \mu+Q^{2}\right)}{Q^{2} \mathrm{C}_{1}(\mu-1)}\right. \\
& \left.\left.-\frac{\mu}{2 \mathrm{C}_{1}^{2} Q^{4}}-\frac{\sqrt{\mu}\left(Q^{2}-4\right)}{Q^{2} \mathrm{C}_{1}}\right)\right],
\end{aligned}
$$

where we have used the relation (85).

\footnotetext{
${ }^{5}$ Notice that (90) is cutoff independent. Therefore this adds a finite contribution to the free energy. However, this boundary term (90) does not have a smooth $Q \rightarrow 0$ limit and is therefore valid only at finite and nonzero $Q$. Also note that in the limit $Q \rightarrow 0$ one can still have a finite contribution provided the temperature is also low $(\sqrt{\mu} \ll 1)$ such that the ratio $\sqrt{\mu} / Q^{2}$ is small but finite.
}

We now analyze the behavior of the free energy of the configuration. In the path integral formulation, the free energy is given by $\Delta \mathcal{F}=-\beta^{-1} \log \mathcal{Z}$, where $\mathcal{Z}$ is the partition function and is defined as ${ }^{6} \mathcal{Z}:=e^{-\Delta S^{(\mathrm{os})}}$. Thus the free energy for the present configuration may be expressed as

$$
\Delta \mathcal{F}:=-\beta_{1}^{-1}\left(-\Delta S^{(\mathrm{os})}\right)
$$

In Fig. 1 we present the behavior of the free energy $\Delta \mathcal{F}$ between the black hole and the interpolating vacuum as a function of the temperature (72). We observe that $\Delta \mathcal{F}<0$ for all values of the temperature $T_{H} \sim \sqrt{\mu}$. The free energy increases asymptotically for sufficiently small values of the temperature and changes its slope at some particular temperature. Afterwards it continues to decrease.

In Fig. 2, we plot the black hole entropy $\mathcal{S}=\mathcal{S}_{W}=$ $-\frac{\Delta \mathcal{F}}{\Delta \sqrt{\mu}}$ against temperature $T_{H} \sim \sqrt{\mu}$. In these plots the entropy is continuous and increases smoothly as we lower the temperature which rules out the possibility of a firstorder phase transition. It is to be noted that this behavior of entropy is consistent with the Wald entropy of the black hole in (73). This allows us to conclude that the phase diagram corresponding to the parameter space with $\mu<1$ is thermodynamically more preferred than that of the $\mu>1$ branch. We comment on the plausible implications of such phase stabilities on the dual SYK physics in the concluding remarks.

\section{EXAMPLE II: EXPONENTIAL COUPLING}

In this section we consider the effective 2D gravity action (1) of the following form:

$$
\begin{aligned}
S_{e d}= & \int d^{2} x \sqrt{-g}\left(\mathcal{R} \Phi^{2}-V\left(\Phi^{2}\right)-\frac{\lambda}{4} e^{\left.-\Phi^{2} F_{\mu \nu} F^{\mu \nu}\right)}\right. \\
& +\int d t \sqrt{-\gamma} \Phi^{2} \mathcal{K},
\end{aligned}
$$

where $\lambda$ is the coupling constant. Also, in our subsequent analysis we shall choose the potential as [37]

\footnotetext{
${ }^{6}$ This definition of the partition function arises from evaluating the path integral for field configurations close to the classical field $\phi_{c l}$ which satisfies the classical equations of motion. If $S[\phi]$ is the corresponding action, then the path integral is dominated by fields $\phi \approx \phi_{c l}+\delta \phi$. Expanding the action for such fields we obtain

$$
S\left[\phi_{c l}+\delta \phi\right] \simeq S\left[\phi_{c l}\right]+\left.\frac{\delta S[\phi]}{\delta \phi}\right|_{\phi_{c l}} \delta \phi+\left.\frac{1}{2} \frac{\delta^{2} S[\phi]}{\delta \phi^{2}}\right|_{\phi_{c l}} \delta \phi^{2}+\cdots
$$

Considering the variation of the fields at the boundary vanishes and neglecting the higher-order terms, the on-shell action is approximately given by $S\left[\phi_{c l}\right]$.
} 

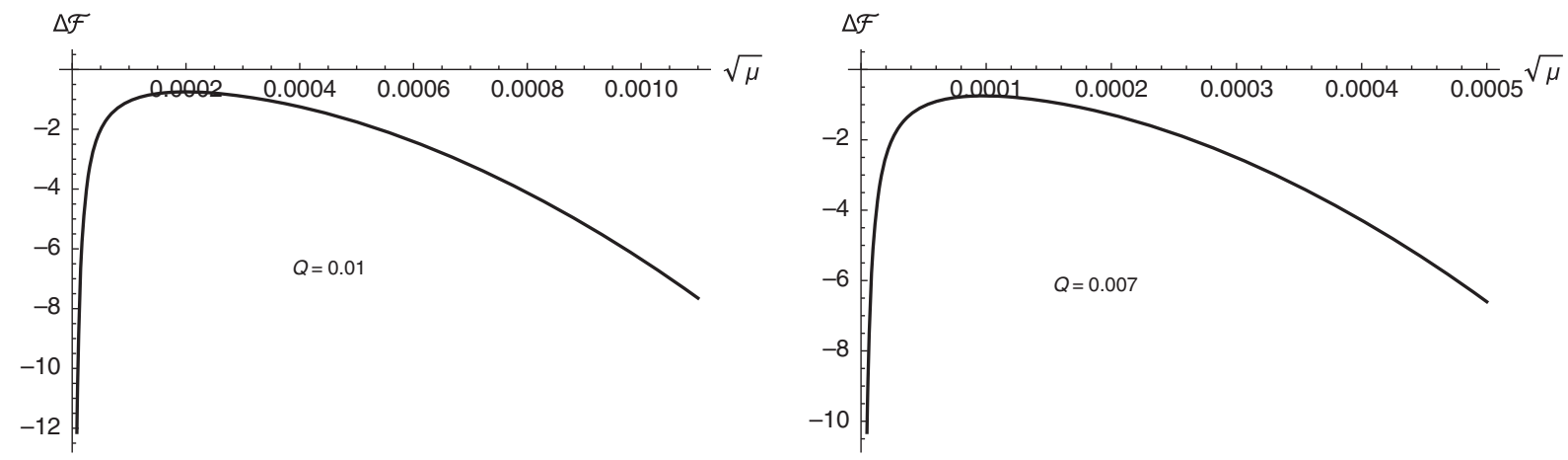

FIG. 1. Free energy of the black hole as a function of the temperature for different values of charge $Q$. We have taken $\mathrm{C}_{1}=1, \mathrm{C}_{2}=0$ and $C=2$
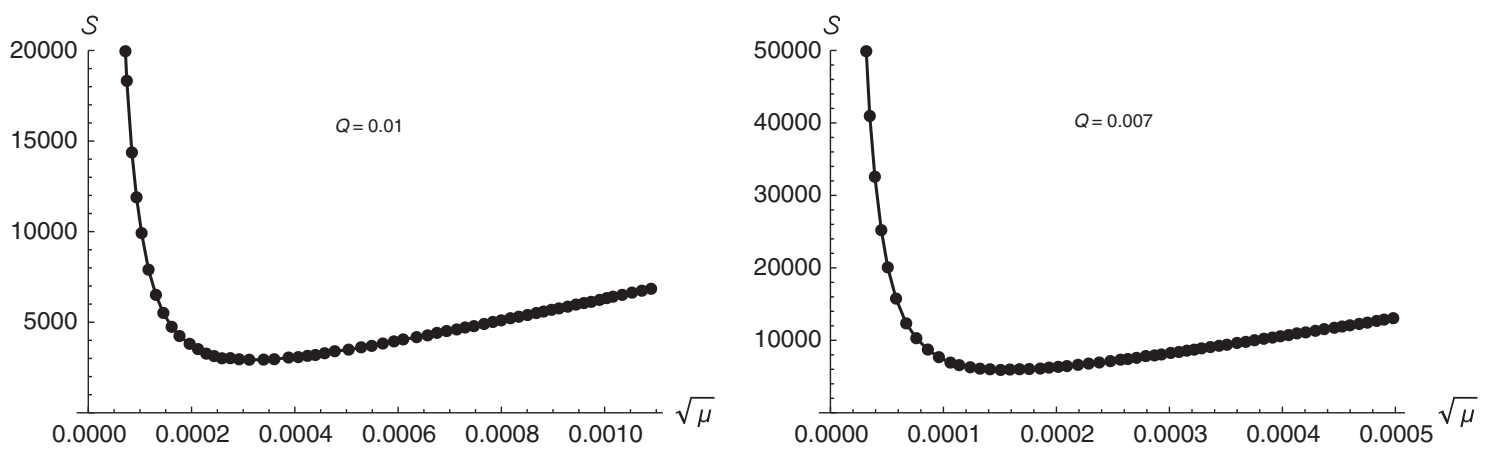

FIG. 2. Thermodynamic entropy $\mathcal{S}=-\frac{\Delta \mathcal{F}}{\Delta \sqrt{\mu}}$ of the black hole as a function of temperature $\sqrt{\mu}$ for different values of charge and $\mathrm{C}_{1}=1, \mathrm{C}_{2}=0$ and $C=2$.

$$
V\left(\Phi^{2}\right)=C-A \Phi^{2}, \quad C, A=2
$$

The corresponding equations of motion are given by

$$
\begin{gathered}
0=\left(\nabla_{\mu} \nabla_{\nu}-g_{\mu \nu} \square\right) \Phi^{2}+\frac{\lambda}{2} e^{-\Phi^{2}}\left(F_{\mu \rho} F_{\nu}{ }^{\rho}-\frac{1}{4} F^{2} g_{\mu \nu}\right) \\
-\frac{1}{2} g_{\mu \nu} V\left(\Phi^{2}\right), \\
0=\mathcal{R}+\frac{\lambda}{4} e^{-\Phi^{2}} F^{2}-\frac{\partial V(\Phi)}{\partial \Phi^{2}}, \\
0=\partial_{\mu}\left(\sqrt{-g} e^{-\Phi^{2}} F^{\mu \nu}\right) .
\end{gathered}
$$

In the next step, using the definition of the metric (4) and the light-cone coordinates $x^{ \pm} \equiv(t \pm z)$ along with the ansatz (6) we rewrite the above equations of motion as

$$
\begin{gathered}
\left(\Phi^{2}\right)^{\prime \prime}+e^{2 \omega} V\left(\Phi^{2}\right)+\frac{\lambda}{2} e^{-\Phi^{2}} e^{-2 \omega} A_{t}^{\prime 2}=0, \\
\left(\Phi^{2}\right)^{\prime \prime}-2 \omega^{\prime}\left(\Phi^{2}\right)^{\prime}=0, \\
2 \omega^{\prime \prime}+e^{2 \omega} \frac{\partial V\left(\Phi^{2}\right)}{\partial \Phi^{2}}+\frac{\lambda}{2} e^{-\Phi^{2}} e^{-2 \omega} A_{t}^{\prime 2}=0,
\end{gathered}
$$

$$
\Phi^{2} A_{t}^{\prime \prime}-A_{t}^{\prime}\left(2 \omega^{\prime}+\left(\Phi^{2}\right)^{\prime}\right)=0 .
$$

The Maxwell field tensor is the solution to (96d) and can be expressed as

$$
F_{z t} \equiv A_{t}^{\prime}(z)=-Q e^{2 \omega} e^{\Phi^{2}} .
$$

Notice that in the absence of charge $(Q)$ the equations of motion correspond to those of the Almheiri-Polchinski model [37]. This allows us to perform a perturbative expansion of the dilaton as well as the metric as done before and find the corresponding vacuum as well as the (charged) black hole solutions.

\section{A. Interpolating vacuum solution}

In the following, we note down the $\mathcal{O}\left(Q^{2}\right)$ equations of motion corresponding to the metric as well as the dilaton:

$$
\begin{aligned}
0= & 2 \omega_{(1)}^{\prime \prime}-4 e^{2 \omega_{(0)}} \omega_{(1)}+\frac{\lambda}{2} e^{2 \omega_{(0)}} e^{\Phi_{(0)}^{2},} \\
0= & \left(\Phi_{(1)}^{2}\right)^{\prime \prime}-2 e^{2 \omega_{(0)}} \Phi_{(1)}^{2} \\
& +4 e^{2 \omega_{(0)}}\left[\omega_{(1)}\left(1-\Phi_{(0)}^{2}\right)+\frac{\lambda}{8} e^{\Phi_{(0)}^{2}}\right],
\end{aligned}
$$

while $\mathcal{O}(1)$ solutions are given by (59) and (60). 
The solution to (98) can be found as

$\omega_{(1)}(z)=\frac{\mathrm{C}_{5}}{z}-\frac{\lambda}{24 z}\left(-2 z e^{1+1 / 2 z}\left(1+2 z-4 z^{2}\right)+e \operatorname{Ei}\left(\frac{1}{2 z}\right)\right)$,

where the exponential integral function is given by

$$
\operatorname{Ei}(z)=-\mathcal{P} \int_{-z}^{\infty} d t \frac{e^{-t}}{t} .
$$

Finally, we express the metric (4) as

$$
d s^{2}=e^{2 \omega_{(0)}(z)}\left(1+2 Q^{2} \omega_{(1)}(z)\right)\left(-d t^{2}+d z^{2}\right) .
$$

The behavior of the metric (4) in the IR $(z \rightarrow \infty)$ is obtained as

$$
\left.e^{2 \omega(z)}\right|_{z \rightarrow \infty} \simeq-\frac{2}{3} e \lambda Q^{2}+\frac{1}{z^{2}}\left(1+\frac{1}{4} e \lambda Q^{2}\right)+\mathcal{O}\left(\frac{1}{z^{3}}\right),
$$

which clearly indicates that the IR behavior of the geometry is $\mathrm{AdS}_{2}$. On the other hand, near the boundary $(z \approx 0)$ the metric (4) behaves as

$$
\left.e^{2 \omega(z)}\right|_{z \approx 0} \simeq \frac{2 Q^{2} \mathrm{C}_{5}}{z^{3}}+\frac{1}{z^{2}}+e^{1 / 2 z} \frac{1}{\mathcal{O}(z)}+\mathcal{O}(1),
$$

whose leading-order term is $\sim 1 / z^{3}$, which is a signature of an asymptotically Lifshitz space-time with dynamical scaling exponent $z_{\text {dyn }}=3 / 2$. Thus the geometry interpolates between $\mathrm{AdS}_{2}$ in the IR and $\mathrm{Lifshitz}_{2}$ in the UV. This observation is similar in spirit to what we have found in the earlier example.

\section{B. Black hole solution}

In order to obtain the charged black hole solution we recall the corresponding zeroth-order solutions (65a) and (65b) and substitute them into (99), which finally yields the first-order correction to the metric:

$$
\begin{aligned}
\omega_{(1)}(\rho)= & \mathrm{C}_{6} \frac{\rho}{\sqrt{\mu}}+\mathrm{C}_{7}\left[-1+\frac{\rho}{\sqrt{\mu}} \cdot \log \sqrt{\frac{\sqrt{\mu}+\rho}{\sqrt{\mu}-\rho}}\right] \\
& -\frac{\lambda e^{1-\sqrt{\mu}}}{8 \mu \sqrt{\mu}}\left[-2 \sqrt{\mu} e^{\rho+\sqrt{\mu}}\right. \\
& +\rho e^{2 \sqrt{\mu}}(\sqrt{\mu}-1) \operatorname{Ei}(\rho-\sqrt{\mu}) \\
& +\rho(\sqrt{\mu}+1) \operatorname{Ei}(\rho+\sqrt{\mu})],
\end{aligned}
$$

where $\mathrm{C}_{6}$ and $\mathrm{C}_{7}$ are arbitrary integration constants and $\operatorname{Ei}(z)$ is given in (101). Notice that in finding the above solution we have used the change in coordinate (67).
Finally, using (51b) we note down the metric (4):

$$
\begin{aligned}
d s^{2} & =e^{2 \omega}\left(-d t^{2}+d z^{2}\right) \\
& =4\left(\rho^{2}-\mu\right)\left(1+2 Q^{2} \omega_{(1)}^{\mathrm{BH}}(\rho)\right)\left(-d t^{2}+\frac{d \rho^{2}}{4\left(\rho^{2}-\mu\right)^{2}}\right) .
\end{aligned}
$$

Clearly, in these coordinates the horizon of the black hole is located at $\rho=\sqrt{\mu}$. On the other hand, the behavior of the metric (106) near the boundary $\rho \rightarrow \infty$ may be found as

$$
\left.e^{2 \omega}(\rho)\right|_{\rho \rightarrow 1 / \Delta} \simeq \frac{e^{1 / \Delta}}{\mathcal{O}(\Delta)}+\frac{8 Q^{2} \mathrm{C}_{6}}{\sqrt{\mu} \Delta^{3}}+\frac{4}{\Delta^{2}}+\frac{1}{\mathcal{O}(\Delta)},
$$

where we have set $\mathrm{C}_{7}=0$ in order to obtain physical boundary conditions in the asymptotic limit of the metric. Referring to (107), we may conclude that the geometry is asymptotically Lifshitz near the boundary of the space-time with dynamical exponent $z_{\mathrm{dyn}}=3 / 2$. This observation is similar to that observed in Sec. V B.

\section{Free energy and phase stability}

In order to understand the underlying phase structure associated with the black hole solution (106) we follow the same line of analysis as in Sec. V C. The on-shell action for the present model may be calculated as

$$
\begin{aligned}
-S_{e d}^{(\mathrm{os})}= & C \int d^{2} x \sqrt{-g} \\
& +\frac{\lambda}{2} \int d^{2} x \partial_{\mu}\left(\sqrt{-g} e^{-\Phi^{2}}\left(1+\Phi^{2}\right) F^{\mu \nu} A_{\nu}\right) \\
& -\frac{\lambda}{2} \int d^{2} x \partial_{\mu}\left(\sqrt{-g} e^{-\Phi^{2}} \Phi^{2} F^{\mu \nu}\right) A_{\nu} \\
& -\int d t \sqrt{-\gamma} \mathcal{K} \Phi^{2} .
\end{aligned}
$$

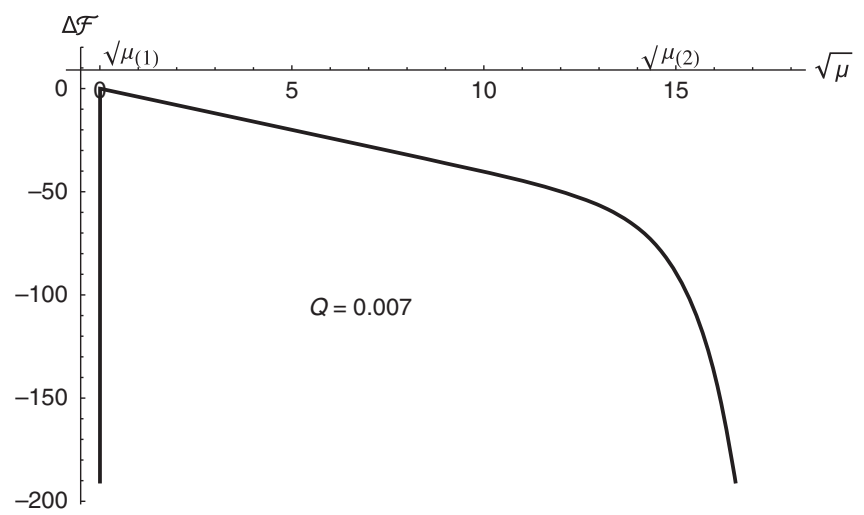

FIG. 3. Free energy of the black hole as a function of the temperature for $Q=0.007$. We have taken $\mathrm{C}_{6}=1$ and $\mathrm{C}_{7}=0$. 

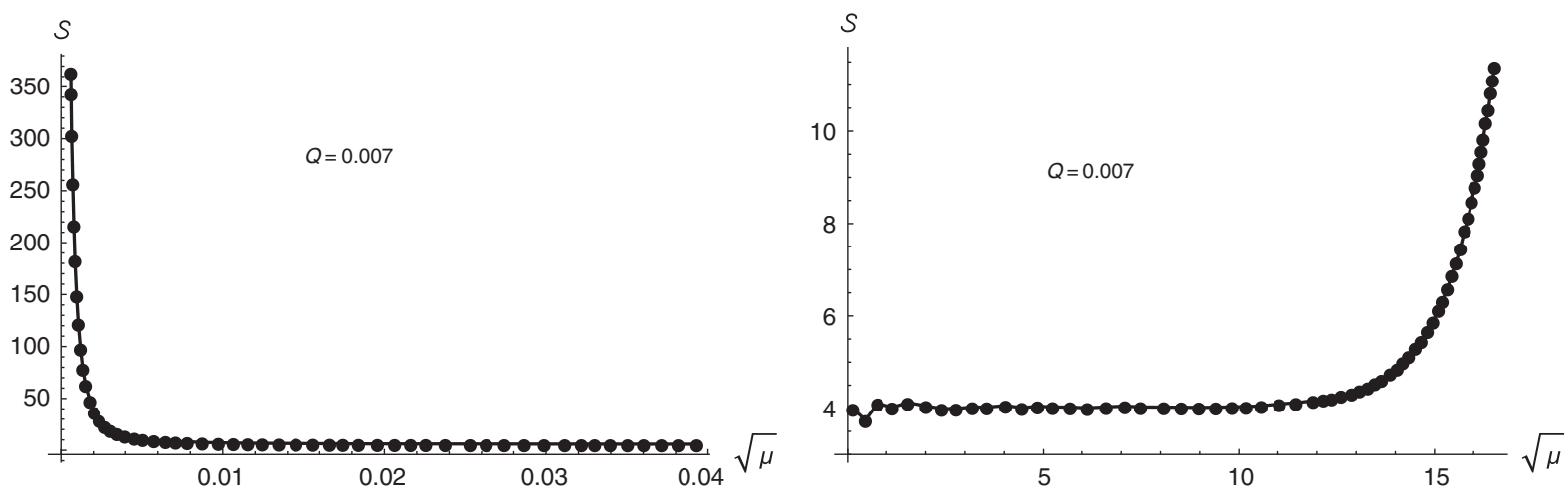

FIG. 4. Thermodynamic entropy $\mathcal{S}=-\frac{\Delta \mathcal{F}}{\Delta \sqrt{\mu}}$ of the black hole as a function of temperature $\sqrt{\mu}$. The left panel corresponds to the left half $[\sqrt{\mu} \in(0,0.04)]$ of the corresponding curve in Fig. 3 while the right panel shows the remaining right half of the same curve. Here $Q=0.007$ and $\mathrm{C}_{6}=1$.

The Abelian 1-form in (108) may be replaced as

$$
A_{t}=-Q \int d z e^{2 \omega_{(0)}} e^{\Phi_{(0)}^{2}}\left(1+\mathcal{O}\left(Q^{2}\right)\right)
$$

where the exponentials in the integrand stand for the zerothorder solutions to the metric and the dilaton.

If we now substitute (97) and (109) into (108), it is easy to check that the second and the third terms in the on-shell action (108) exactly cancel each other. On the other hand, the difference in the volume terms may be schematically expressed as

$$
\begin{aligned}
\Delta(\mathrm{Vol}) & =C\left((\mathrm{Vol})^{\mathrm{BH}}-(\mathrm{Vol})_{\mathrm{Int}}^{\mathrm{vac}}\right) \\
& =-C \beta_{1}\left[\mathcal{D}_{1}-\mathcal{D}_{2}^{(\sqrt{\mu})}-\mathcal{D}_{3} \frac{\beta_{0}}{\beta_{1}}\right],
\end{aligned}
$$

whose finite contribution may be expressed as

$$
\mathcal{D}_{2}^{(\sqrt{\mu})}=\frac{1}{2 \sqrt{\mu}}\left[e^{1+\sqrt{\mu}} Q^{2} \lambda+4 \mu\left(1+\mathrm{C}_{6} Q^{2}\right)\right]
$$

Finally, using the definition (92) the corresponding free energy can be expressed as ${ }^{7}$

$$
\Delta \mathcal{F}=-\frac{1}{\pi}\left[e^{1+\sqrt{\mu}} Q^{2} \lambda+4 \mu\left(1+\mathrm{C}_{6} Q^{2}\right)\right],
$$

where $\beta_{1} \equiv 1 / T_{H}=\pi / \sqrt{\mu}$ is the inverse Hawking temperature of the black hole, which is easily calculated by substituting the metric components of (106) in (72).

We now plot the free energy $(\Delta \mathcal{F})$ of the black hole against the temperature $T_{H} \sim \sqrt{\mu}$ (see Fig. 3). Furthermore in Fig. 4, we show the changes in entropy $\mathcal{S}=S_{W}$ $(=-\Delta \mathcal{F} / \Delta \sqrt{\mu})$ against the temperature $T_{H} \sim \sqrt{\mu}$. From

\footnotetext{
${ }^{7}$ For the present model, the Gibbons-Hawking-York boundary term in (108) does not provide any finite contribution to the free energy.
}

this plot it is evident that there is no first-order phase transition as the $\mathcal{S}-\sqrt{\mu}$ plot is continuous. We further observe that this behavior of entropy is consistent with that of the Wald entropy of the corresponding black hole which can be easily computed using (51a) and (54) and is given by

$$
S_{W}=4 \pi\left[1+\sqrt{\mu}+Q^{2} \frac{\lambda e^{1+\sqrt{\mu}}+4 \mathrm{C}_{6} \mu}{4 \sqrt{\mu}}\right] .
$$

From Fig. 3 we notice two turning points, $\sqrt{\mu}_{(1)}$ and $\sqrt{\mu}_{(2)}\left(\sqrt{\mu}_{(1)}<\sqrt{\mu}_{(2)}\right)$, at which the sign of the slope changes. This behavior is reflected in the corresponding entropy plots in Fig. 4 in which the entropy increases asymptotically both for $\sqrt{\mu}<\sqrt{\mu}_{(1)}$ and $\sqrt{\mu}>\sqrt{\mu}_{(2)}$. In the window $\Delta \sqrt{\mu} \sim \sqrt{\mu}_{(2)}-\sqrt{\mu}_{(1)}$ the entropy does not change considerably. In this window the system falls in the minimum entropy state whose plausible interpretation in terms of SYK degrees of freedom are discussed in the concluding remarks.

\section{CONCLUDING REMARKS}

In the present paper, we have proposed models of charged solutions within the framework of $1+1 \mathrm{D}$ JT gravity. Based on our model computations, we have shown that in the presence of nontrivial couplings between the $U(1)$ gauge field and the dilaton the asymptotic geometries get substantially modified both for the vacuum as well as the black hole solutions. In both the examples, the vacuum solutions interpolate between Lifshitz $_{2}$ in the UV to $\mathrm{AdS}_{2}$ in the IR. On the other hand, the black hole solutions turn out to be asymptotically Lifshitz $z_{2}$ with $z_{\text {dyn }}=3 / 2$.

We have further analyzed the stability of black holes in both the models and observed a universal feature in the free energy and therefore the entropy of the system. In the first model we have considered the quadratic coupling and in the second model the exponential coupling of the dilaton to the 
gauge field. In both the cases, at sufficiently low temperatures, we observed a turning point after which the free energy falls off sharply (Figs. 1 and 3) which corresponds to an increase in the entropy below this temperature (Figs. 2 and 4).

The existence of minimal entropy at low temperatures could be interpreted as the formation of the Bose-Einsteinlike condensate ${ }^{8}$ (BEC) in the dual SYK model which possibly leads toward superfluid instabilities at low temperatures and finite density [67]. We hope to clarify some of these issues from the perspective of the dual SYK physics in the near future.

\section{ACKNOWLEDGMENTS}

The work of A.L. is supported by the Chilean FONDECYT Project No. 3190021. D. R. is indebted to the authorities of IIT Roorkee for their unconditional support toward researches in basic sciences. Both the authors would like to thank Hemant Rathi, Jitendra Pal and Dr. Arup Samanta for useful discussions. Special thanks to Jakob Salzer for his valuable comments on the manuscript.

\section{APPENDIX A: A NOTE ON DIMENSIONAL REDUCTION}

In this section, we propose a dimensional reduction procedure in order to show that our $1+1 \mathrm{D}$ dilaton gravity models (2) and (93) are indeed effective models of a higherdimensional gravity theory.

Let us consider the following $2+1 \mathrm{D}$ Einstein-dilaton gravity:

$S_{3 \mathrm{D}}=\int d^{3} x \sqrt{-g^{(3)}}\left(\mathcal{R}^{(3)}+\chi(\Psi)+\gamma\left(\partial_{z} \Psi\right)\left(\partial^{z} \Psi\right)\right)$,

where $\mathcal{R}^{(3)}$ is the three-dimensional Ricci scalar and $\chi(\Psi)$ is the dilaton potential which includes the cosmological constant as we see below. Here $\Psi \equiv \Phi^{2}$ of the original analysis.

In order to obtain a $1+1 \mathrm{D}$ effective action, we dimensionally reduce (A1) along the compact direction $\theta$ :

$$
d s_{(3)}^{2}=d s_{(2)}^{2}+e^{-2 \beta \Psi}\left(d \theta+\tilde{A}_{a} d x^{a}\right)^{2},
$$

where $d s_{(2)}^{2}$ is the usual $\mathrm{AdS}_{2}$ metric (4) with $e^{2 \omega}=1 / z^{2}$.

Notice that in (A2) the indices $a, b$ run over the uncompactified directions. Also, we have identified $\theta \sim$ $\theta+2 \pi$ and assumed an $U(1)$ symmetry for the dilaton

\footnotetext{
${ }^{8}$ Notice that the classical solutions in the JT gravity correspond to large $N$ dynamics in the dual SYK model. Therefore, one should interpret the charged condensate as a classical (large $N$ ) analog of the BEC-like phenomena [62-66] in the dual SYK picture where quantum fluctuations are suppressed because of $1 / N$ corrections.
}

field $\Psi$. The gauge fields $\tilde{A}_{a}$ in (A2) are known as the Kaluza-Klein vectors. In the subsequent analysis, we choose to work with the ansatz (6).

In the next step, we wish to calculate the $2+1 \mathrm{D}$ Ricci scalar $\mathcal{R}^{(3)}$ which is related to the $1+1 \mathrm{D}$ Ricci scalar $\mathcal{R}^{(2)}$ as

$\mathcal{R}^{(3)}=\mathcal{R}^{(2)}-\frac{1}{4} e^{-2 \beta \Psi} \tilde{F}_{\mu \nu}^{2}+2 \beta\left(\partial_{z} \partial^{z} \Psi\right)-2 \beta^{2}\left(\partial_{z} \Psi\right)\left(\partial^{z} \Psi\right)$.

We now find a relation between the determinants of the two metrics as

$$
\sqrt{-g^{(3)}}=\sqrt{-g^{(2)}} e^{-\beta \Psi} .
$$

If we now substitute (A3) and (A4) in (A1), the $2+1 \mathrm{D}$ action reduces to the following $1+1 \mathrm{D}$ form:

$$
S_{2 \mathrm{D}}=\int d^{2} x \sqrt{-g^{(2)}} e^{-\beta \Psi}\left[\mathcal{R}^{(2)}+\chi(\Psi)-\frac{1}{4} e^{-2 \beta \Psi} \tilde{F}_{\mu \nu}^{2}\right],
$$

where we set $\beta= \pm \sqrt{\gamma / 2}$ and $\gamma=2$.

If we redefine $\Psi \rightarrow \log \Psi$ and use $\beta=-1$ in (A5) we recover an action which is similar in spirit to the action (2) corresponding to model I. Notice that, in order to obtain the desired form of the potential, one must set $\chi=(A-C / \Psi)$, where $A$ plays the role of cosmological constant in the original $2+1 \mathrm{D}$ gravity model (A1). On the other hand, in the limit $\Psi \ll 1$ we obtain an action similar to (93) which corresponds to that of model II. In this case we set $\beta=-1$ and $\chi \approx-C+(C+A) \Psi$.

\section{APPENDIX B: BLACK HOLE SOLUTION WITH $A<0$}

In this Appendix, we discuss the metric solution corresponding to our first model (2) while considering the linear dilaton potential as $U\left(\Phi^{2}\right)=C+A \Phi^{2}$. In order to simplify the calculations we choose $A, C=2$.

Using the perturbation expansion (51a) and (51b), we write down the equation of motion (7c) up to leading order in the expansion as

$$
\mathcal{O}\left(Q^{0}\right): 0=2 \omega_{(0)}^{\prime \prime}(z)+2 e^{2 \omega_{(0)}(z)}
$$

$\mathcal{O}\left(Q^{2}\right): 0=2 \omega_{(1)}^{\prime \prime}(z)+4 e^{2 \omega_{(0)}(z)} \omega_{(1)}(z)-\frac{e^{2 \omega_{(0)}(z)}}{\left(\Phi_{(0)}^{2}\right)^{3}}$.

Similarly, substituting (51a) and (51b) in the dilaton equation of motion (7a) we obtain 


$$
\begin{gathered}
\mathcal{O}\left(Q^{0}\right): 0=\left(\Phi_{(0)}^{2}\right)^{\prime \prime}+2 e^{2 \omega_{(0)}}\left(1+\Phi_{(0)}^{2}\right), \\
\mathcal{O}\left(Q^{2}\right): 0=\left(\Phi_{(1)}^{2}\right)^{\prime \prime}+2 e^{2 \omega_{(0)}} \Phi_{(1)}^{2} \\
+4 e^{2 \omega_{(0)}}\left[\omega_{(1)}\left(1+\Phi_{(0)}^{2}\right)+\frac{1}{8}\left(\Phi_{(0)}^{2}\right)^{-2}\right] .
\end{gathered}
$$

The zeroth-order equations (B1) and (B3) have the following solutions:

$$
\begin{gathered}
e^{2 \omega_{(0)}}=\frac{4 \mu}{\cosh ^{2} 2 \sqrt{\mu} z}, \\
\left(\Phi_{(0)}^{2}\right)=-(1+\sqrt{\mu} \tanh 2 \sqrt{\mu} z) .
\end{gathered}
$$

Notice that, in obtaining the solution (B6), we have set $\bar{C}=-1$ and $\tilde{C}=-\frac{1}{2}$ in (54).

Now, using (51a) and (51b) in (B2), the leading-order solution $\omega_{(1)}^{\mathrm{BH}}$ can be expressed as

$$
\begin{aligned}
\omega_{(1)}^{\mathrm{BH}}(\rho)= & \frac{\rho \mathrm{C}_{8}}{\sqrt{\mu}}+\mathrm{C}_{9}\left[-1+\frac{\rho}{\sqrt{\mu}} \tanh ^{-1}\left(\frac{\rho}{\sqrt{\mu}}\right)\right]+\frac{1}{8 \mu-1^{2} \mu^{3 / 2}(1+\rho)} \\
& \times\left\{-2 \sqrt{\mu}(\mu-1)(-1+\mu-\rho)+\rho(1+\rho)\left[4 \mu^{3 / 2} \cdot \log (1+\rho)\right.\right. \\
& \left.\left.+\left(-1+3 \mu-2 \mu^{3 / 2}\right) \log (\rho-\sqrt{\mu})+\left(1-3 \mu-2 \mu^{3 / 2}\right) \log (\rho+\sqrt{\mu})\right]\right\},
\end{aligned}
$$

where $\mathrm{C}_{8}$ and $\mathrm{C}_{9}$ are constants of integration. In writing (B7) we have made the following change in the spatial coordinate:

$$
z \rightarrow \frac{1}{2 \sqrt{\mu}} \tanh ^{-1}\left(\frac{\rho}{\sqrt{\mu}}\right) .
$$

Finally, using (51b) and (B5), the metric (4) corresponding to the black hole can be written as

$$
d s^{2}=4\left(\mu-\rho^{2}\right)\left(1+2 Q^{2} \omega_{(1)}(\rho)\right)\left(-d t^{2}+\frac{d \rho^{2}}{4\left(\mu-\rho^{2}\right)^{2}}\right) .
$$

Clearly, the horizon of the black hole (B9) is located at $\rho=\sqrt{\mu}$. However, from the structure of the solution (B5) the position of the boundary of the space-time (B9) is not quite apparent.

The Hawking temperature corresponding to the above black hole (B9) can be written down using the formula (72) and is given by

$$
T_{H}=\frac{\sqrt{\mu}}{2 \pi}
$$

The corresponding Wald entropy can be expressed as

$$
S_{W}=4 \pi\left[1+\sqrt{\mu}+Q^{2} \frac{1+4 \mathrm{C}_{8} \mu|\mu-1|}{4 \sqrt{\mu}|\mu-1|}\right] .
$$

Notice that, in writing (B11), we have used (51a) and (54).
[1] J. M. Maldacena, The large $N$ limit of superconformal field theories and supergravity, Int. J. Theor. Phys. 38, 1113 (1999); Adv. Theor. Math. Phys. 2, 231 (1998).

[2] E. Witten, Anti-de Sitter space and holography, Adv. Theor. Math. Phys. 2, 253 (1998).

[3] O. Aharony, S. S. Gubser, J. M. Maldacena, H. Ooguri, and Y. Oz, Large $N$ field theories, string theory and gravity, Phys. Rep. 323, 183 (2000).

[4] S. Sachdev and J. Ye, Gapless Spin Fluid Ground State in a Random, Quantum Heisenberg Magnet, Phys. Rev. Lett. 70, 3339 (1993).

[5] S. Sachdev, Holographic Metals and the Fractionalized Fermi Liquid, Phys. Rev. Lett. 105, 151602 (2010).
[6] S. Sachdev, Strange metals and the AdS/CFT correspondence, J. Stat. Mech. (2010) P11022.

[7] A. Kitaev, A simple model of quantum holography, in Proceedings of the KITP Strings Seminar and Entanglement Program, February 12, April 7, and May 27, Santa Barbara, CA, USA (2015).

[8] A. Kitaev, Hidden correlations in the Hawking radiation and thermal noise, in Proceedings of the Fundamental Physics Prize Symposium, November 10, Santa Barbara, CA, USA (2014).

[9] S. Sachdev, Bekenstein-Hawking Entropy and Strange Metals, Phys. Rev. X 5, 041025 (2015).

[10] J. Polchinski and V. Rosenhaus, The spectrum in the SachdevYe-Kitaev model, J. High Energy Phys. 04 (2016) 001. 
[11] J. Maldacena and D. Stanford, Remarks on the Sachdev-YeKitaev model, Phys. Rev. D 94, 106002 (2016).

[12] W. Fu, D. Gaiotto, J. Maldacena, and S. Sachdev, Supersymmetric Sachdev-Ye-Kitaev models, Phys. Rev. D 95, 026009 (2017); 95, 069904(E) (2017).

[13] J. Yoon, Supersymmetric SYK model: Bi-local collective superfield/supermatrix formulation, J. High Energy Phys. 10 (2017) 172.

[14] A. M. Garcia-Garcia and J. J. M. Verbaarschot, Spectral and thermodynamic properties of the Sachdev-Ye-Kitaev model, Phys. Rev. D 94, 126010 (2016).

[15] A. M. Garcia-Garcia and J. J. M. Verbaarschot, Analytical spectral density of the Sachdev-Ye-Kitaev model at finite $N$, Phys. Rev. D 96, 066012 (2017).

[16] A. Jevicki, K. Suzuki, and J. Yoon, Bi-local holography in the SYK model, J. High Energy Phys. 07 (2016) 007.

[17] A. Jevicki and K. Suzuki, Bi-local holography in the SYK model: Perturbations, J. High Energy Phys. 11 (2016) 046.

[18] D. J. Gross and V. Rosenhaus, A generalization of SachdevYe-Kitaev, J. High Energy Phys. 02 (2017) 093.

[19] D. J. Gross and V. Rosenhaus, The bulk dual of SYK: Cubic couplings, J. High Energy Phys. 05 (2017) 092.

[20] A. Kitaev and S. J. Suh, The soft mode in the Sachdev-YeKitaev model and its gravity dual, J. High Energy Phys. 05 (2018) 183.

[21] C. Krishnan, S. Sanyal, and P. N. Bala Subramanian, Quantum chaos and holographic tensor models, J. High Energy Phys. 03 (2017) 056; M. Berkooz, P. Narayan, M. Rozali, and J. Simon, Higher dimensional generalizations of the SYK model, J. High Energy Phys. 01 (2017) 138.

[22] C. Peng, M. Spradlin, and A. Volovich, Correlators in the $\mathcal{N}=2$ supersymmetric SYK model, J. High Energy Phys. 10 (2017) 202.

[23] C. Peng, M. Spradlin, and A. Volovich, A supersymmetric SYK-like tensor model, J. High Energy Phys. 05 (2017) 062 .

[24] M. Taylor, Generalized conformal structure, dilaton gravity and SYK, J. High Energy Phys. 01 (2018) 010.

[25] S. Forste, J. Kames-King, and M. Wiesner, Towards the holographic dual of $N=2 \mathrm{SYK}$, J. High Energy Phys. 03 (2018) 028.

[26] V. Rosenhaus, An introduction to the SYK model, arXiv: 1807.03334.

[27] T. Ishii, S. Okumura, J. I. Sakamoto, and K. Yoshida, Gravitational perturbations as $T \bar{T}$-deformations in 2D dilaton gravity systems, arXiv:1906.03865.

[28] D. J. Gross, J. Kruthoff, A. Rolph, and E. Shaghoulian, $T \bar{T}$ in $\mathrm{AdS}_{2}$ and quantum mechanics, arXiv:1907.04873.

[29] A. M. Charles and F. Larsen, A one-loop test of the near-AdS $\mathrm{A}_{2} /$ near-CFT 1 correspondence, arXiv:1908.03575.

[30] U. Moitra, S. K. Sake, S. P. Trivedi, and V. Vishal, Jackiwteitelboim gravity and rotating black holes, J. High Energy Phys. 11 (2019) 047.

[31] U. Moitra, S. K. Sake, S. P. Trivedi, and V. Vishal, Jackiwteitelboim model coupled to conformal matter in the semiclassical limit, arXiv:1908.08523.

[32] C. Teitelboim, Gravitation and hamiltonian structure in two space-time dimensions, Phys. Lett. B 126, 41 (1983).

[33] R. Jackiw, Lower dimensional gravity, Nucl. Phys. B252, 343 (1985).
[34] D. Grumiller, W. Kummer, and D. V. Vassilevich, Dilaton gravity in two-dimensions, Phys. Rep. 369, 327 (2002).

[35] D. Grumiller and R. McNees, Thermodynamics of black holes in two (and higher) dimensions, J. High Energy Phys. 04 (2007) 074.

[36] D. Grumiller, R. McNees, and J. Salzer, Cosmological constant as confining $\mathrm{U}(1)$ charge in two-dimensional dilaton gravity, Phys. Rev. D 90, 044032 (2014).

[37] A. Almheiri and J. Polchinski, Models of $\mathrm{AdS}_{2}$ backreaction and holography, J. High Energy Phys. 11 (2015) 014.

[38] J. Maldacena, D. Stanford, and Z. Yang, Conformal symmetry and its breaking in two dimensional nearly Anti-deSitter space, Prog. Theor. Exp. Phys. 2016, 12C104 (2016).

[39] M. Cvetic and I. Papadimitriou, $\mathrm{AdS}_{2}$ holographic dictionary, J. High Energy Phys. 12 (2016) 008; Erratum, J. High Energy Phys. 01 (2017) 120(E).

[40] G. Mandal, P. Nayak, and S. R. Wadia, Coadjoint orbit action of Virasoro group and two-dimensional quantum gravity dual to SYK/tensor models, J. High Energy Phys. 11 (2017) 046.

[41] J. Engelsoy, T. G. Mertens, and H. Verlinde, An investigation of $\mathrm{AdS}_{2}$ backreaction and holography, J. High Energy Phys. 07 (2016) 139; K. Jensen, Chaos in $\mathrm{AdS}_{2}$ Holography, Phys. Rev. Lett. 117, 111601 (2016).

[42] S. R. Das, A. Jevicki, and K. Suzuki, Three dimensional view of the SYK/AdS duality, J. High Energy Phys. 09 (2017) 017.

[43] S. R. Das, A. Ghosh, A. Jevicki, and K. Suzuki, Three dimensional view of arbitrary $q$ SYK models, J. High Energy Phys. 02 (2018) 162.

[44] S. R. Das, A. Ghosh, A. Jevicki, and K. Suzuki, Space-time in the SYK model, J. High Energy Phys. 07 (2018) 184.

[45] H. Kyono, S. Okumura, and K. Yoshida, Deformations of the Almheiri-Polchinski model, J. High Energy Phys. 03 (2017) 173.

[46] H. Kyono, S. Okumura, and K. Yoshida, Comments on 2D dilaton gravity system with a hyperbolic dilaton potential, Nucl. Phys. B923, 126 (2017).

[47] S. Okumura and K. Yoshida, Weyl transformation and regular solutions in a deformed Jackiw-Teitelboim model, Nucl. Phys. B933, 234 (2018).

[48] A. Lala and D. Roychowdhury, SYK/AdS duality with YangBaxter deformations, J. High Energy Phys. 12 (2018) 073.

[49] D. Roychowdhury, Holographic derivation of $q$ SYK spectrum with Yang-Baxter shift, Phys. Lett. B 797, 134818 (2019).

[50] R. A. Davison, W. Fu, A. Georges, Y. Gu, K. Jensen, and S. Sachdev, Thermoelectric transport in disordered metals without quasiparticles: The Sachdev-Ye-Kitaev models and holography, Phys. Rev. B 95, 155131 (2017).

[51] A. Gaikwad, L. K. Joshi, G. Mandal, and S. R. Wadia, Holographic dual to charged SYK from 3D Gravity and Chern-Simons, arXiv:1802.07746.

[52] E. Witten, Anti-de Sitter space, thermal phase transition, and confinement in gauge theories, Adv. Theor. Math. Phys. 2, 505 (1998).

[53] J. W. York, Jr., Role of Conformal Three Geometry in the Dynamics of Gravitation, Phys. Rev. Lett. 28, 1082 (1972).

[54] G. W. Gibbons and S. W. Hawking, Action integrals and partition functions in quantum gravity, Phys. Rev. D 15, 2752 (1977). 
[55] M. Natsuume, AdS/CFT Duality User Guide, Lecture Notes in Physics, Vol. 903, English ed. (Springer, Heidelberg, 2015).

[56] C. G. Callan, Jr., S. B. Giddings, J. A. Harvey, and A. Strominger, Evanescent black holes, Phys. Rev. D 45, R1005 (1992).

[57] R. C. Myers, Black hole entropy in two-dimensions, Phys. Rev. D 50, 6412 (1994).

[58] E. D'Hoker and P. Kraus, Magnetic brane solutions in AdS, J. High Energy Phys. 10 (2009) 088.

[59] F. Apruzzi, M. Fazzi, A. Passias, D. Rosa, and A. Tomasiello, $\mathrm{AdS}_{6}$ solutions of type II supergravity, J. High Energy Phys. 11 (2014) 099; 05 (2015) 12.

[60] M. Taylor, Non-relativistic holography, arXiv:0812.0530.

[61] E. Witten, Anti-de Sitter space, thermal phase transition, and confinement in gauge theories, Adv. Theor. Math. Phys. 2, 505 (1998).
[62] K. Staliunas, Bose-einstein condensation in classical systems, http://xxx.lanl.gov/abs/cond-mat/0001347 (2000).

[63] K. Stalinas, Bose-Einstein condensation in dissipative systems far from thermal equilibrium, arXiv:cond-mat/ 0001436.

[64] O. V. Utyuzh, G. Wilk, and Z. Wodarczyk, New proposal of numerical modelling of Bose-Einstein correlations: BoseEinstein correlations from within, arXiv:hep-ph/0511309.

[65] A. A. Ezhov and A. Yu. Khrennikov, Agents with left and right dominant hemispheres and quantum statistics, Phys. Rev. E 71, 016138 (2005).

[66] O. Utyuzh, G. Wilk, and Z. Wlodarczyk, Modelling bose einstein correlations via elementary emitting cells, Phys. Rev. D 75, 074030 (2007).

[67] D. R. Tilley and J. Tilley, Superfluidity and Superconductivity, 3rd ed. (Institute of Physics Publishing Ltd., Bristol, 1990). 